Wiktor Grygorenko

\title{
DESIGN ELEMENTS OF GEOGRAPHICAL MAP CONTENTS
}

The process of making maps (mapping, charting) represents in itself a particular method of analysis and of the conscious (purposeful) processing of information about events taking place inside the geographical space. Details emerging during the process of mapping, apart from giving a cartographic record of some fragment of the space under study, are above all a medium of information well-founded scientifically and based on the surveying and experiment and fulfilling requirements of the cartographic convention. In map contents we can find information about characteristic land configuration (relief), about spatial relationships, as well as information about certain properties and correlations existing between natural objects and phenomena within the geographical space under study.

A map, to be brief, constitutes a geometrical and symbolic information model of geographical realities. Such a definition emphasizes two constructional peculiarities of map contents. The first one applies to geometrical structure of the cartographic image, the other one to the cartographic symbolism. Under the term "geographical structure of the cartographic image" we understand the layout and positioning of the signs that designate objects and phenomena entering into the composition of map contents in a strictly defined geometrical manner. Under the term "cartographic symbolism", on the other hand, we understand the system of cartographic signis which on the map perform the role of representatives of the field objects, their characteristics, or relations existing between them so as to enable the viewer to understand the sense of separate fragments and to perceive map contents as a whole, too.

One of the basic requirements to be met by geometrical structure of a cartographic image is that actual differentiation of objects and phenomena observed in various fragments of the geographical space should find its reflection in graphic presentation of the density with which 
symbols do appear in relevant parts of the map. It is only then that the cartographic model is likely to offer a chance of revealing similarities and differences in respective fragments of the geographic space. Graphic contrast between individual parts of the map will apply not only to the external features, i.e. dimensions, geometry of layout, and the number of cartographic signs, but to the internal features, i.e. logics and causalities as well as component elements of the objects and phenomena having their expression in the complexity of drawing and in the colour of cartographic signs, as well.

In addition to the specific character the differentiation of the geographic space may exhibit, geometrical structure of the cartographic image hides in itself also genesis of the geographical phenomena which in order to be learnt require that organization of the spatial links and of the way in which map content components have been arranged ought to be studied in detail. A map fulfills therefore the role of an excellent tool (instrument) rendering it possible to investigate thoroughly and to learn at the same time geographical realities. Research aiming at the disclosure of structural regularities in the construction of map contents has led cartographers to the conclusion that each map, no matter how specific this technical product may be, represents above all an authentic piece of the work. Each map, as a matter of fact, accounts for a certain synthetic amount of our overall knowledge about the Earth and about phenomena occurring in it, and as such it represents yet another step forward towards the fuller familiarization with objects and phenomena around us, and towards progress of the scientific thought.

As a product of scientific thought and as a technical achievement at the same time, each map is liable to some more general laws, i.e. rules of scientific and technological design and is expected to meet certain specific requirements of the production technology.

When planning map contents and form of the graphic image, i.e. when considering the substance and the "symbolic" side of the process, use will have to be made of uniform, quantitative, design parameters of the cartographic information. Quantitative parameters may be referred to as factors featuring the particular type of the map we are having to do with, and as elements decisive for the characteristic usefulness of the geographic map. In their number we can find, for instance, coefficients of composition and norms of the map-content generalization, the rate of cartographic image surface density, rate of concentration of the cartographic signs, and the size of signs. 
Long years of cartographic experience corroborate the fact that quantitative design of geographical map contents exhibits all symptoms of a universal process according to which maps of any type and on any level of minuteness of detail, matched with the actual map application, can be constructed.

Hence, any object of design, and maps or yet more precisely map contents are no exception to this rule, may be regarded as a sort of system in which strict correlation exists between all component elements. From the point of view of the part these elements are expected to perform in the said system, they may be possibly divided into two groups: one - essential, component or design, elements of an object; two - supplementary elements

In this division, design elements are the most meaningful as they prove to be independent of, or only slightly dependent on, the remaining ones. On the other hand, all the supplementary elements usually remain to a high degree, or even completely, dependent on the design elements.

The map, as a geometrical and symbolic model of geographical reality, transmits information in the form of a graphic record. The drawing with its cartographic signs shows how objects and natural phenomena in the area under study are related versus each other and what are their characteristics, and may be quoted as a good example of such a record.

In the case of maps, the theme and the application of a map both embodied in the idea of the map itself; projection and scale; the $\mathrm{sy}^{\mathrm{r}}$ stem of cartographic signs for identifying objects and phenomena represented; all these constitute map-content design elements.

We are in a position to distinguish seven stages in the process of scientific and technical design of map content (Fig. 1).

Stage I-Formulation of a general idea of map content; explicitness of purpose and application of the map; indication of source materials for use in the mapping process; delimitation of presumable circles of map users as well as prospective ways or scope of map uses.

Stage II-Thematical study of the map and investigation of the map area for its geographical features.

Stage III-Scrutiny of source materials;

Stage IV-Specification of the basic map-content design parameters:

a) Selection and computation of cartographic projection;

b) Fixing the percentage of map-content components (p-natural, a-anthropogenic, t-technical) and wording of the content-generalization norms; 


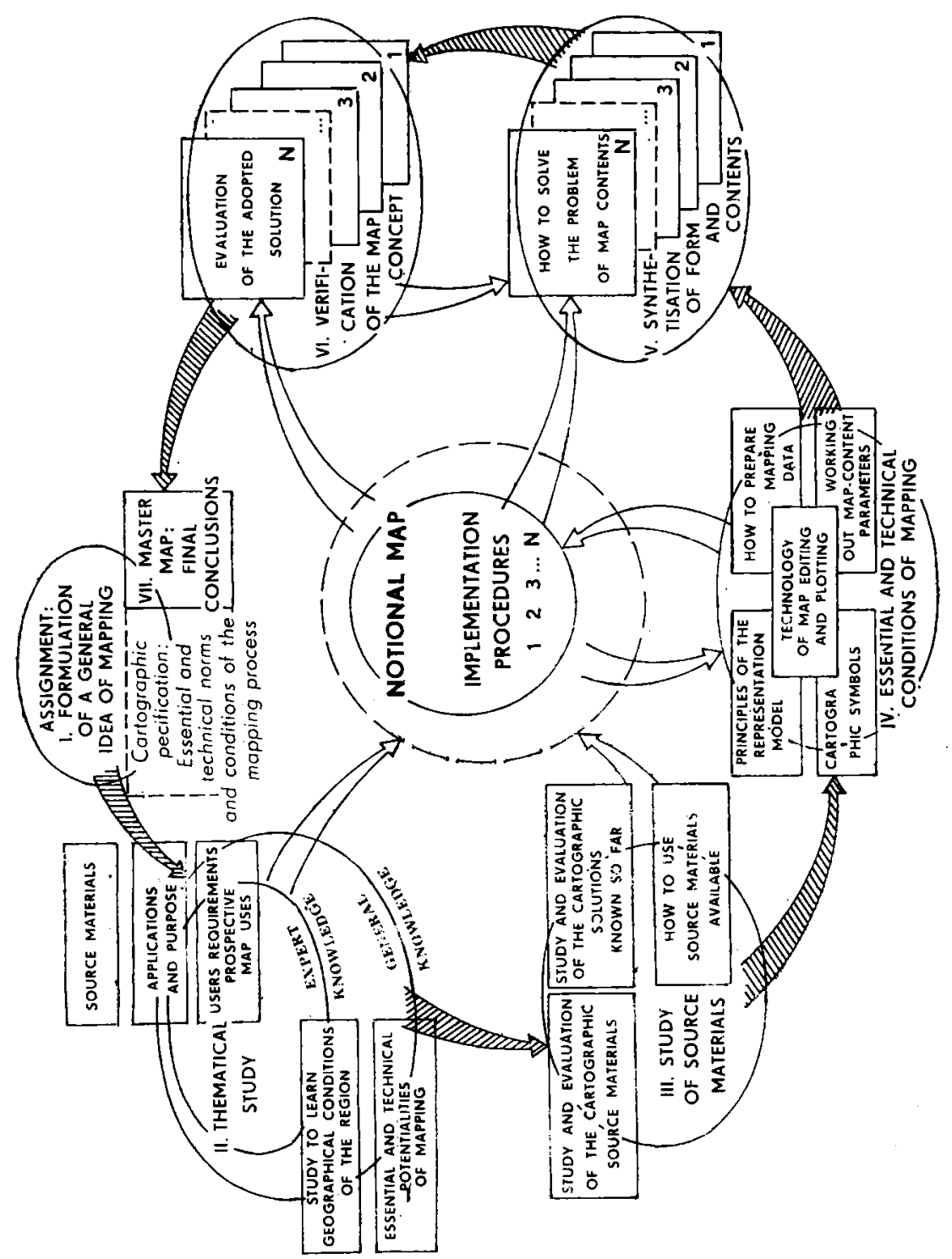

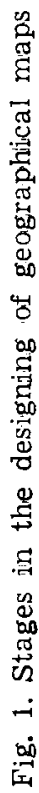


c) Elaboration of a system of cartographic signs.

Stage V-Preparation of the master-map.

Stage VI-Verification and evaluation of the master-map for its compliance with the previously-established conditions applying to essentials and technicalities. Approval or projection of the proposed solution.

Stage VII-Drafting of operational standards and conditions for the implementation of the approved master-map.

The scheme of scientific and technical design of map content as presented in this paper includes all stages followed normally under a routine mapping process known to the cartographers. It focuses, moreover, our attention on the problem of the so-called notional map as an intermediate link between relevant stages of the map-content designing process.

The first idea of the map to be plotted emerges in the author's mind as soon as he gets information that such and such a map is necessary. As the map desigining stages advance in their succession, an increasing number of details is made available and on their basis an abstract concept of map comes to fruition, materializing in a number of the master maps (Stage V). As a result, the map under preparation reaches the stage of practical accomplishment and a final decision is taken as to the share respective components are expected to have in it and as to the form of presentation.

As far as contents and form of the map are concerned (the essence of the process), these must be worked out in full compliance with the substantial and technical, quantitative, parameters of the map content, laid down well in before. Substantial parameters normally include: cartographic map projection, coefficients of map-content composition, generalization norms, informative power of the cartographic code. Technical parameters, on the other hand, include surface density of signs and the size of cartographic signs used in the mapping process.

Quantitative map-content parameters are of theoretical and technological importance only. They .will also decide about the effectiveness of the map in functioning as an information medium, and about the informative value of such a map. They also indicate which of the map-designing stages may be found most suitable for formal treatment.

While considering the informative value of a map, we are often inclined to make use of the notion of completeness or fullness of contents. Both these terms lack no doubt precision for a complete set of information will each time be defined by the final objective a map has been designed to serve. Moreover, the multitude and varsatility of details concerning field objects plotted are so great that it becomes im. 
possible to supply all of them on one map only, whatever universal it may be. This implies the necessity of producing a number of maps differing from each other in the amount of details, scale, and in the degree of synthesizing, i.e. in the processing of information plotted.

While speaksing about processing of the cartographic information one must have in mind the degree of generality with which such an information has been digested to produce it on the map. Accordingly, we may have to do with a completely or partly processed, or totally unprocessed, cartographic information. The greatest interpretation potentialities, i.e. the greatest stock of information about geographical space as a whole and about its component elements, are offered by the unprocessed information in the sheer descriptive form, as e.g. information furnished by the large-scale topographic maps (Fig. 2). Information that has been completely processed (Fig. 3) lacks such a merit for it becomes well disputable, if not completely futile sometimes, to draw conclusions other than those stated on the map.

It is possible to process cartographic information by way of reduction or aggregation, or by way of division and integration.

The process of reduction includes purposeful selection and elimination of certain details of map contents, featuring lower informative power. The process of aggregation, on the other hand, consists in more general notions being given to the details. For instance, instead of saying "Leafy Forest" or "Coniferous Forest" a more general term "Woodland" may be introduced. Procedures like this find their practical expression when making maps more general in their geographical character, topographic maps in the first place. In consequence, a certain grading takes place in the amount of details being plotted.

The process of division and integration consists mainly in the isolation of information on separate components of the map content, or even on single objects or phenomena being shown on the map with their individualistic and technical specifications given in a more or less general form. This process is usually accompanied by a change in the system of cartographic signs from the viewpoint of their informative qualities and graphic construction. Processing of information in such a way will be characteristic of the procedures connected with the editing of thematical maps.

Processes of reducing, aggregating, dividing and integrating cartographic information serve the purpose of simplifying cartographic description of the geographical space. The above-mentioned procedures represent, in principle, diverse forms of generalization operations in the cartographic practice. They may be framed in the form of mathemati- 

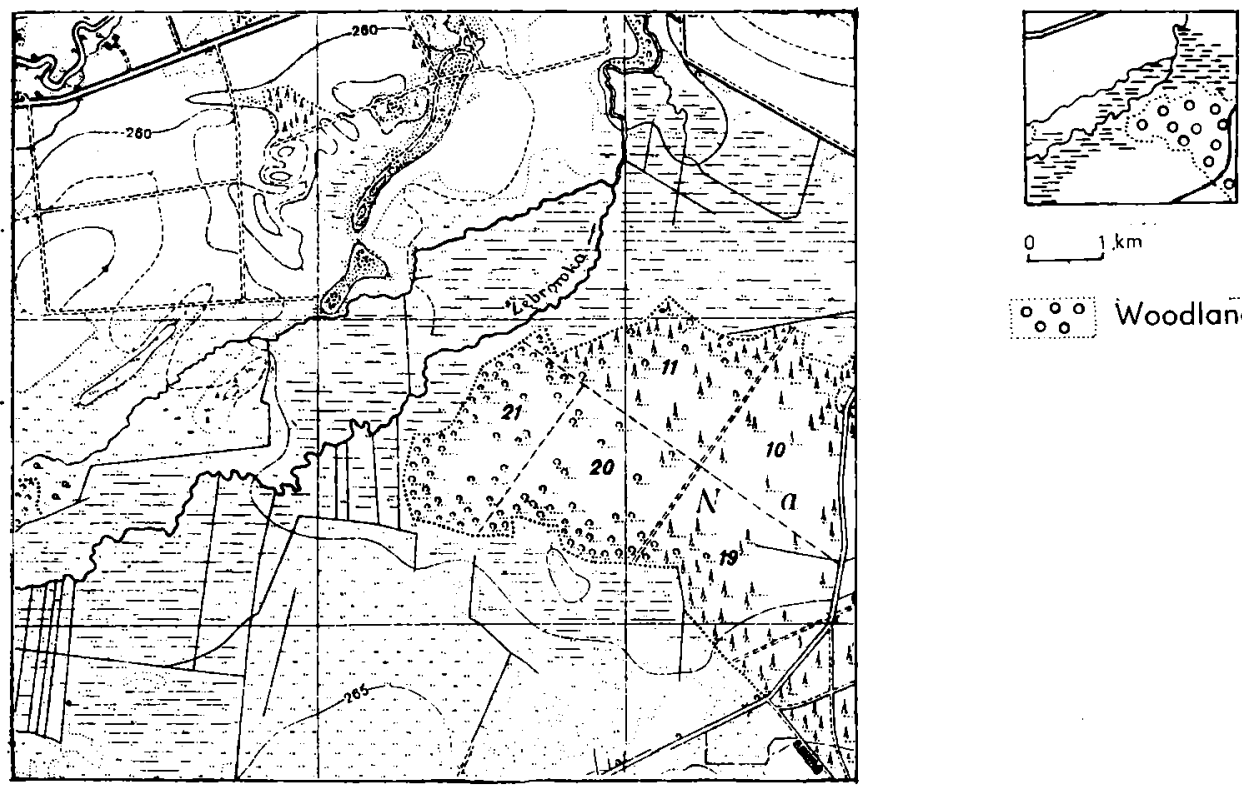

\%o Woodland

0 $1 \mathrm{~km}$

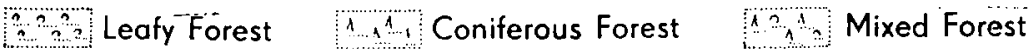

Fig. 2. Topognaphical map - straightforward, unprocessed information
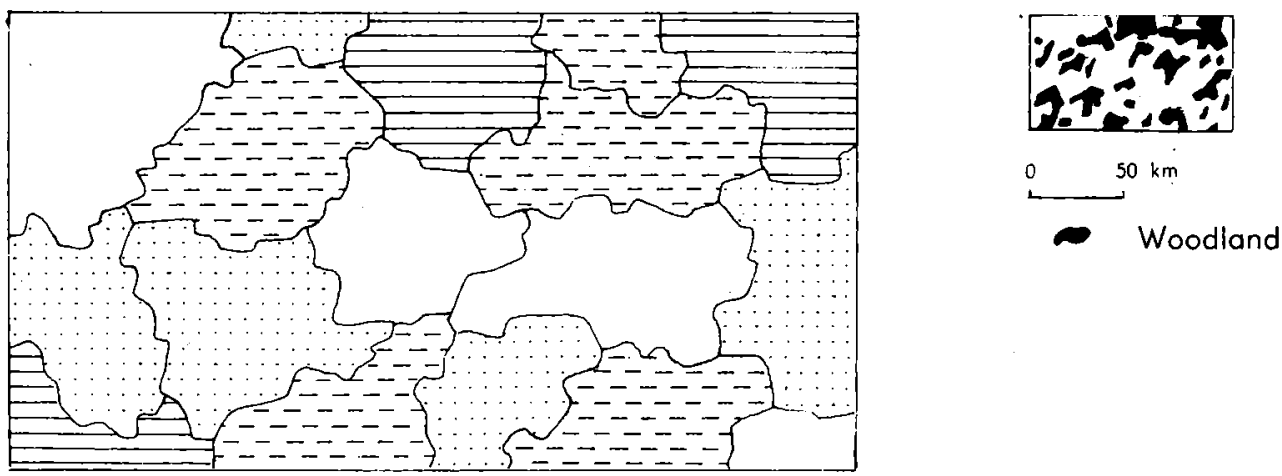

0

$50 \mathrm{~km}$

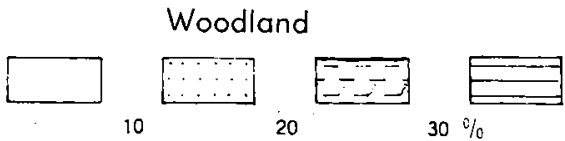

Fig. 3. Cartogram - totally processed complex information 
cal formulae to enable formalization of operations in the process of editing and plotting the content of maps.

As it is known, each fragment of the geographic space may differ a lot from the other ones in the composition of objects and geographical phenomena found therein. To reproduce this diversification on the map appears to be the main objective of a mapping process. Map content must therefore be designed so as to convey features characteristio of the respective regions; in other words, adequate graphic technique has to be adopted in onder to plot objects and phenomena falling under the following three independent systems: Natural Phenomena (P), Anthropogénic Phenomena (A), Ontogenetic and Technical (T) Features of the Natural and Anthropogenic Phenomena (Ontogenetic and technical features of the natural and anthropogenic phenomena can be approached to as thematical component of map content).

Graphic elements of map contents whose concentration (i.e. quantitative density) or surface density within the area of map field under study exceeds certain predetermined level are typical of such a field, whereas other elements constitute only a "background". Such a formalization of features of the map field under study will enable us to use mathematical methods in calculating quantitative parameters of contents for each field of the map.

To express typological characteristics of a field use can be made, for instance, of composition coefficients of the main map components according to the formulae:

$$
p=\frac{g_{\mathrm{P}}}{m^{\prime} K_{\mathrm{P}}} ; \quad a=\frac{g_{\mathrm{A}}}{m^{\prime} K_{\Lambda}} ; \quad t=\frac{g_{\mathrm{T}}}{m^{\prime} K_{\mathrm{T}}} .
$$

In these formulae, letters $p, a, t$ denote composition coefficients of the characteristic map components: P - Natural, A - Anthropogenic, T Technical:

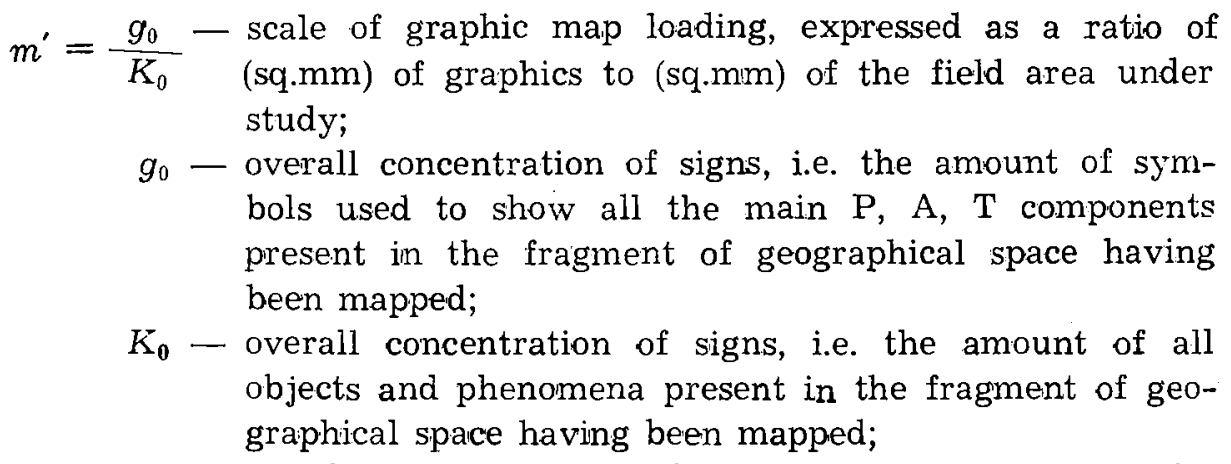

$g_{\mathrm{P}}, g_{\mathrm{A}}, g_{\mathrm{T}}-$ specific concentration of signs on the map, i.e. the 
amount of signs in the main $\mathrm{P}, \mathrm{A}, \mathrm{T}$ component collections in the map field under study;

$K_{\mathrm{P}}, K_{\mathrm{A}}, K_{\mathrm{T}}-$ specific concentration of objects and phenomena, i.e. the amount of objects and phenomena, natural $\mathrm{P}$, anthropogenic $A$, as well as ontogenetic and technical peculiarities $\mathrm{T}$, in the fragment of geographic space covered by the map field under study.

Composition coefficients $\mathrm{p}, \mathrm{a}, \mathrm{t}$ of the map contents indicate in which proportions basic components $\mathrm{P}, \mathrm{A}, \mathrm{T}$ of the geographic reality are expected to be found in the cartographic image of the map field under study. With their help we can calculate the degree of concentration of signs corresponding to the main components in each field of the map, with due proportions being maintained between them in the horizontal plane, i.e. between all the fields, and in the vertical one, i.e. between main components in each of the fields.

Coefficients of map-content composition therefore perform also the role of uniform norms for the quantitative generalization of contents in all scales of the general geographical maps. 
